\title{
Un eslabón en el recorrido estético de Stravinski: la Suite italiana
}

\author{
Beatriz Luis Moreno \\ bea.luis.moreno@gmail.com \\ Centro Superior Katarina Gurska
}

\section{Resumen}

Igor Stravinski se erige como una de las figuras más destacadas de la música del siglo XX, y es también uno de los compositores que más importancia le ha concedido al pensamiento estético y filosófico en materia musical. Se ha perseguido dar una explicación más detallada a preguntas relacionadas con la naturaleza del fenómeno musical recogidas en sus libros, así como a otros aspectos diferentes que trata el compositor en relación con la figura del intérprete o del compositor director de sus propias obras. Hemos llevado la investigación a un ámbito más práctico, a través, principalmente, de un análisis de recursos y materiales, así como diferencias entre las partituras del ballet Pulcinella y de la Suite italiana.

\section{Palabras Clave}

Stravinski, estética, Suite Italiana, fenómeno musical, Dushkin 


\section{Aesthetics in Stravinski: Italian Suite}

\section{Abstract}

Igor Stravinski stands as one of the most remarkable figures in music of the 20th century and he is one of the composers who has given more importance to aesthetics and philosophical thinking in the field of music. We wanted to take a look into his whole aesthetic thinking, comparing all the sources given by the composer himself. Our aim is to give a more detailed explanation to some questions related to the nature of the musical phenomenon that are included in his books, as well as other different aspects the composer deals with in relation to the figure of the performer or the composer-conductor of his own compositions. We have taken this research to a more practical level through an analysis mainly of resources and materials, as well as the differences in the Pulcinella ballet and the Italian Suite scores.

\section{Keywords:}

Stravinski, aesthetics, Italian Suite, musical phenomenon, Dushkin

\section{Concepción Estética de Stravinski}

La reflexión filosófica y estética adquiere gran importancia en la figura de Igor Stravinski, estando estrechamente ligadas a sus etapas compositivas y desarrollándose con ellas. Para él, estas reflexiones y el concepto del estilo musical son inseparables, evolucionando conjuntamente (Ferrer, 2012, p. 3).

Stravinski (1936) le da gran importancia a la composición sobre un instrumento, en su caso el piano, ya que considera que es indispensable componer en contacto con la materia sonora. Le otorga también un gran peso a la improvisación como método de exploración e imaginación creadora. Como reflejo de este pensamiento, señala que es posible percibir cuándo un compositor compone música de un instrumento o para este, ya que se ve reflejado en la calidad sonora del resultado. Insiste también en que el compositor debe sentir las vibraciones, no solo tocar (Manufacturing Intellect, 2020). Además, considera que es de vital importancia que el compositor sea también director de sus propias obras, ya que de esta manera puede tener una mejor comprensión del funcionamiento de la orquesta. Gracias a ello, se adquiere una mayor calidad de escritura que refleja exactamente los propósitos individuales.

Más centrado en su etapa neoclásica (1920-1951), que comienza con el estreno de Pulcinella, reflexiona sobre el motor de la composición, preguntándose si es 
el respeto o el amor hacia el pasado lo que le lleva a componer dichas obras. La cuestión concluye constatando que para componer hace falta un motor que nos impulse, por lo tanto, el respeto quedaría relegado a un segundo plano al no dar frutos. Destaca la voluntad como un factor fundamental. No se puede catalogar la sonoridad como disonante, ya que los diferentes estratos que componen las obras son consonantes, dando como resultado la audición de diferentes funciones armónicas simultáneamente (De la Motte, 1995).

Así mismo, recalca en los directores de orquesta el rigor con el que traten el material sonoro, evitando el afecto o connotaciones subjetivas de cualquier tipo y ciñéndose exclusivamente a lo que esté escrito en la partitura.

Respecto al papel que desempeña el ejecutante en el proceso musical, Stravinski expresa criterios muy definidos. El compositor sitúa esta figura como eje indispensable para la transmisión de la música, destacando la importancia del movimiento y los gestos para que el contenido musical se manifieste íntegramente. Esto ocurre siempre que dichos movimientos fluyan acordes a la obra que se esté ejecutando, sin exageraciones propias del sentimentalismo y la subjetividad. Así mismo, el ejecutante debe ser capaz de captar lo que hay escrito en la partitura, sin añadir nada más. Por lo tanto, decreta claramente que su función es transmitir la música, no interpretarla. Dicho pensamiento queda recogido en Stravinsky. Ideas y Recuerdos: "[l]a ejecución o exhibición de ejecución se ha desarrollado hasta tal punto que está desafiando a la música misma, e incluso amenaza con relegarla a un puesto secundario" (Craft, 1971, p. 17).

Stravinski pone de manifiesto la importancia de dicho ejecutante, entendido como intermediario entre el compositor y su público. De él depende la opinión que tendrá el público sobre su obra, hecho que no ocurre en otras artes como la pintura o la escultura, en las cuales dicho intermediario no existe. Sin embargo, podemos encontrar un ejemplo similar en el teatro, donde el actor es también una pieza de unión entre la obra escrita y sus receptores, y de su actuación dependerá que llegue bien el mensaje y que el público la acepte. De esta manera, tanto el actor como el ejecutante juegan un papel crucial en el acto comunicativo que supone el arte, ya que sin estas figuras dicho acto no se completaría. El teatro y la música necesitan del dinamismo para transmitirse íntegramente.

Romeu señala que "la creación del arte se divide en dos momentos básicos: la concepción que es imaginativa, y la conformación que es su materialización. El discurso se encuentra desperdigado en ambos; el mensaje artístico, en cambio, es sólo parte del último" (2007, p. 3). De esta afirmación podemos deducir que el mensaje como tal solo se da en la conformación del arte, es decir que, en el caso de la música, únicamente lo encontraríamos en el momento que el intérprete ejecuta la obra, aunque el discurso lo encontremos previamente en la partitura que 
nos ha dejado el compositor. Dicha conformación se da cuando la obra causa una reacción en el espectador, dejando ver su contenido comunicativo (Ruiz Molina, 2018).

No obstante, para Stravinski la figura del ejecutante se disipa cuando es él mismo quien la asume o quien dirige sus obras, como es el caso del contrato discográfico con Columbia. A raíz de este, podemos observar una reflexión sobre la difusión y el fácil acceso a la música gracias a los discos y otros medios. Por un lado, lo ve como un hecho positivo ya que permite que la música llegue a una mayor cantidad de personas. Por otro, lo considera bastante negativo, ya que conlleva a que estemos constantemente expuestos a música y que, por lo tanto, no tengamos que hacer ningún esfuerzo activo por escucharla: "[s]obresaturados de sonidos, las gentes caen en una suerte de embrutecimiento que les quita toda facultad de discernimiento y les vuelve indiferentes a la calidad misma de la música que se les sirve" (Stravinski, 1936, p. 161). Además, contempla la mentira que supone la grabación, ya que la ejecución de una pieza es un hecho vivo y que debe ser contemplado en directo.

Sin embargo, considera que la relación entre el compositor y el público es compleja, ya que usualmente no existe una comunicación clara entre ambos. Esto lleva a malentendidos, porque cuando el compositor muestra su personalidad normalmente es rechazado y poco comprendido. En numerosas ocasiones, Stravinski se percibe a sí mismo por delante del pensamiento del público, lo que dificulta la comunicación y causa una mala comprensión del mensaje sonoro.

Cabe destacar también la opinión que tiene el compositor acerca de los instrumentos de arco. En un primer momento, llega a rechazarlos por su poca precisión y articulación en comparación con los instrumentos de viento. No obstante, en torno a la composición de Apolo musageta vuelven a retomar importancia en su lenguaje compositivo, destacando en ellos su aproximación al canto y sus características melódicas. De esta manera observamos en el compositor una vuelta al cultivo de la melodía.

\section{Opinión de la Sociedad Contemporánea}

Stravinski critica profundamente la sociedad de su época, ya que la encuentra predecible y llena de contradicciones. Por un lado, señala la tendencia al ateísmo, que se enfrenta a su admiración por León Tolstoi, de espíritu cristianizante. Es también un momento en el que se aboga mucho por el naturalismo y el espíritu folclórico, aunque el público no rechazaba el sinfonismo alemán de Johannes Brahms y Richard Wagner.

Respecto a los compositores contemporáneos a Stravinski, podemos destacar, por un lado, al grupo encabezado por Nikolái Rimski-Korsakov y Aleksandr 
Glazunov, al que señala como academicista y seguidor de dogmas que él mismo decide aceptar. Por otro lado, con el descubrimiento de autores franceses como Claude Debussy, se da cuenta de que dichos dogmas pueden verse alterados, y es por ello que reflexiona acerca de la unión entre la estética y la técnica, que define como inseparables: "toda doctrina estética tiene necesidad de una manera de expresión propia y, por tanto, de una técnica propia, pues no puede concebirse un arte sin una técnica que no derive de un fondo estético determinado" (Stravinski, 1936, p. 30).

En relación con Wagner, resalta la impresión que Stravinski nos ofrece sobre Parsifal. Sin negar la calidad de esta música, se sorprende negativamente al notar que ha pretendido llevarla al mismo plano que el servicio religioso, lo que considera errado por la propia actitud con la que se acude a ambos lugares. Considera que el auditor va a juzgar lo que escucha, mientras que la persona que acude al servicio religioso no juzga, ya que se guía por la fe. De esta manera, no es concebible la equiparación de ambos. Por lo general, la oposición al pensamiento wagneriano es un aspecto destacado en su estética.

En cuanto al ballet, tan relevante en su vida, era ampliamente menospreciado en la época, ya que la sociedad lo veía como un género inferior. No obstante, gracias a Mijaíl Fokin y a señalados bailarines de la época, experimentó un importante auge. Stravinski pone de manifiesto su admiración por el ballet clásico, que se caracteriza por la gran importancia del orden y las reglas. El compositor ve en esta danza la oposición entre lo apolíneo y lo dionisíaco, entendiendo como apolíneo aquello más relacionado con la estructura y la forma, mientras que lo dionisíaco se relaciona con el dinamismo y lo sublime. Encuentra en la danza clásica la máxima expresión de lo apolíneo, lo que la hace digna de admiración.

La distinción entre lo dionisíaco y lo apolíneo había tenido especial importancia en el romanticismo gracias a la figura de Friedrich Nietzsche y su relación con Wagner. Sin embargo, en este periodo se comprende dicha distinción como elemento unificador de las artes, derivado de la tragedia griega, mientras que para Stravinski será lo contrario. Stravinski no perseguirá la unión de las artes como habían hecho los compositores afines a Wagner. De hecho, considera el drama wagneriano un invento que ha hecho decaer a la cultura musical, reduciéndola al nivel de cansar al oyente y hacer que este no disfrute. Así, muestra su posición claramente formalista acusando a Wagner de haber acabado con la forma musical a causa del drama creado por dicho compositor y considerando el concepto de obra de arte total como algo dañino y no fundamental.

Cabe señalar que Stravinski en ningún momento niega a Wagner como un compositor excepcional. Él defiende que tanto en la música como en las demás artes existe una jerarquía en las formas pertinentes. Así, le concede más impor- 
tancia a la música instrumental que a la vocal al estar esta última intrínsecamente vinculada a la palabra. Las distintas características de los diferentes tipos de música dan lugar a gran variedad de formas, siendo la sinfonía la más completa y rica. No obstante, señala que la música vocal, por el hecho de estar ligada a la palabra, ha entrado en decadencia.

Podemos señalar también la importancia que tiene para Stravinski poseer un conocimiento activo y profundo de la actualidad, ya que considera que sin él no se pueden abordar épocas pasadas. Este pensamiento queda recogido en Crónicas de mi vida: "[p]ues solo aquellos que están esencialmente vivos saben descubrir la vida real en los que están 'muertos"' (Stravinski, 1936, p. 86). Este conocimiento, unido a una cultura musical profunda, es necesario también en los ejecutantes. El compositor apunta que es una cualidad subestimada en los considerados virtuosos, que persiguen el éxito y la sensación, dejando de lado usualmente dicho conocimiento.

\section{Concepción de la Música}

$\mathrm{Al}$ contrario que los compositores románticos, Stravinski concibe la música como un fenómeno incapaz de cualquier expresión, incluso cuando parece incuestionable esta cualidad, la considera una quimera. Destaca la música como construcción capaz de dotar de orden y tiempo, que es capaz de producir una emoción equiparable a la contemplación de obras arquitectónicas. Considera la música como un fin, consecuencia de la ordenación de materiales por parte del ser humano. Dicha concepción se deriva del pensamiento de Arthur Schopenhauer, para el cual los sonidos están presentes en el universo y el ser humano los traduce y ordena, construyendo para ello nuevos objetos.

Como vemos, Stravinski defiende la música como un fenómeno fruto de la especulación, la cual actúa como su energía creadora. En ella se organizan sonido y tiempo para dar lugar a obras musicales. Necesita de la existencia de un valor que pueda ser medido, el metro, el cual compone el ritmo (Olives, 2004). De esta manera, podemos observar en él una visión mucho más objetiva y de índole científica, producto claro del formalismo de Eduard Hanslick, que explicaremos a continuación.

\section{El Formalismo de Hanslick}

Con anterioridad a Hanslick existen ya algunos filósofos que esbozan las ideas de lo que más tarde se convertiría en el formalismo, hecho que supuso el comienzo del fin del romanticismo. Enrico Fubini sentencia: "Hanslick viene a ser el anti-Wagner por excelencia, la primera reacción violenta y radical que se fragua contra el romanticismo, contra la concepción de la música como expresión de 
sentimiento o de cualquier otro contenido" (2005, p. 343). Con dicha afirmación posiciona claramente a Hanslick como el eje central de todo este movimiento, ya que asienta los cimientos que darán forma al pensamiento de músicos más o menos contemporáneos.

Para comprender mejor el punto de partida del formalismo, debemos conocer que, aun no estando descrito ni creado el concepto de musicología ni, por tanto, profesionalizada la figura del musicólogo como tal, Hanslick supuso desde un comienzo una novedad. Dicha novedad se debe a que las críticas musicales que realizó durante su vida son claramente el producto de una gran concisión y un profundo conocimiento técnico, por lo que podemos presuponer su profundo conocimiento musical.

Su formalismo se deriva principalmente del pensamiento de Johann Herbart e Immanuel Kant, defendiendo y reelaborando el arte como forma y no como expresión, justo al contrario de lo que se había defendido tan estoicamente durante el romanticismo. De esta manera, se comprende que lo emotivo o expresivo no debe tomarse como punto de referencia para determinar la calidad de una obra artística, al ser esto profundamente subjetivo y al no ser determinante para dicha obra.

$\mathrm{Al}$ aceptar la forma como elemento definitorio y clave del arte, se está asimilando también que cada una de las disciplinas tendrá características diferentes y, por tanto, se contempla una visión individualista de cada una de ellas. Asume cada una de ellas como diferentes y no comparables bajo los mismos espectros, por lo que no podemos establecer cuál de ellas es superior o inferior a las demás. Fubini expresa:

(...) como base de esta afirmación, se halla el concepto fundamental del pensamiento de Hanslick: la identificación de la música con su técnica. La técnica musical ya no es un medio para expresar sentimientos, para conocer lo absoluto o para suscitar emociones; es exclusivamente la música, nada más (2005, p. 345).

Aquí podemos observar cómo la corriente definida por Hanslick está eminentemente fundamentada en la objetividad prácticamente científica, en la que priman las actitudes derivadas del análisis. Aboga por un acercamiento que presupone similar al que se trabaja en las ciencias naturales. No obstante, nunca llega a negar que la música pueda tener una influencia y relación con los sentimientos, sino que más bien relega esto a un plano más secundario y menos importante a la hora de determinar cuál es el valor de las obras. Lo principal y más importante es la forma, ya que no podemos distinguirla del contenido, porque todo es forma y esta es un elemento completo que no necesita de otros para consumarse. 
Sin embargo, tenemos que destacar que Hanslick no niega por completo la relación de la música con el aspecto emotivo y sentimental. Como él mismo explica, la música no está completamente alejada del mundo de los sentimientos, ya que representa su dinamismo y movimiento (Fubini, 2005). Con este enunciado queremos dar a entender que la música describe cómo podemos sentir la emoción y las fases que esta tiene, pero no la emoción propiamente dicha. No obstante, defiende la existencia de una jerarquía entre la música determinada por los sentimientos y la determinada por las formas, siendo más relevante la segunda, digna de contemplación. De este modo, solo los que tienen la capacidad de contemplar la forma pueden acceder a la belleza musical (Imberty, 2001).

Así mismo, podemos comprender esta corriente desde la concepción de la música como actividad cognitiva, al ser "sensible, pues su ámbito es exclusivamente el sonido; pero de algún modo, es también intelectual" (Díaz Soto, 2009, p. 177). Con este enunciado se plantea la visión contraria a la emotividad como parte fundamental y definitoria de la música, y se pone el énfasis en la parte objetiva, intelectual, es decir, lo relacionado con la técnica y la forma esencialmente.

Por último, cabe destacar la definición de la música como arte carente de la semántica que posee el lenguaje, estableciendo así una clara distinción. Esta clasificación se debe principalmente a la imposibilidad de traducir la música a dicho lenguaje. La diferencia también radica en la importancia del sonido en ambos, ya que, en el lenguaje, el sonido se comporta como un simple medio por el cual se transmiten los mensajes, y en la música el sonido es fundamental y goza de una gran importancia.

\section{Desarrollo del Pensamiento de Stravinski}

Como podemos apreciar en su Poética Musical, el pensamiento estético de Stravinski está abiertamente influenciado por el de Pierre Souvtchinski, al que considera un referente, aunque, como hemos mencionado se deriva directamente del formalismo de Hanslick. Stravinski otorga una gran importancia a la dimensión temporal de la música, entendiéndose esta como una organización del tiempo. Esta concepción de la música deriva del pensamiento de Souvtchinski, quien la divide en dos categorías que recogen esencialmente todo lo expuesto en corrientes estéticas anteriores.

Para Souvtchinski existe, por una parte, la música cronoamétrica, que es la caracterizada por ser capaz de mostrar las emociones y es atemporal, siendo Wagner, Liszt, Berlioz o Rimski-Korsakov ejemplos de compositores inscriptos en esa categoría. Por otra parte, existe la música cronométrica, que es definida por ser "siempre ontológica, y se caracteriza por la ausencia de movimientos psicológicos” (Ares Yebra, 2017, p. 154). 
Esta música cronométrica es en la que se inscriben tanto Stravinski como Debussy, y en ella la importancia radica en el desarrollo de la obra en un tiempo definido como ilimitado y único. Con esta división podemos observar que para Souvtchinski, la dimensión temporal en la música es algo fundamental y definitorio, y por lo tanto también para Stravinski, ya que su pensamiento deriva directamente, como decíamos, del pensamiento de este autor, cuya teoría considera afín a sus propias concepciones. Sin embargo, se puede considerar que Stravinski, al aplicar dicha organización temporal a sus materiales, limita la evolución de los mismos (Kaiero, 2007).

De esta definición de la música como organización, en primer lugar, nace la crítica a Wagner precisamente por su carencia. Para el compositor ruso, el orden y la disciplina son indispensables, mientras que considera la música de Wagner como fruto de la improvisación y, por lo tanto, carente de tal orden. El principal punto de crítica respecto a lo anterior se sitúa en la melodía infinita asumida por Wagner, la cual Stravinski considera "un ultraje a la dignidad y a la función misma de la melodía, que es, ya lo hemos dicho, el canto musical de una frase cadenciada" (Stravinski, 1946, p. 85). De esta manera, podemos ver que para Stravinski este tipo de melodía no tiene motivo para ser creada, lo cual supone una contradicción directa con el objetivo wagneriano y una crítica a Wagner precisamente por la falta de disciplina.

Por otro lado, Stravinski (1936) resalta la necesidad de restricciones y normas a la hora de componer, considerándolas necesarias para poder hacer uso de la libertad. Para él, sin la existencia de normas no hay orden ni lirismo. Compara el trabajo del compositor con el de un artesano, que trabaja bajo encargos, pero realiza obras de arte.

De esta concepción de la música como una organización del tiempo se deriva también la idea de la existencia de polos de atracción dentro de ese espacio temporal. Stravinski desliga estos polos de cualquier técnica compositiva, pero su imparcialidad es falsa ya que el hecho de definirlos como factores que implican atracción se deriva claramente del pensamiento tonal que se ha cultivado a través de la historia. Además, también considera la melodía como la parte fundamental del discurso musical, según la cual se rigen el tiempo y el reposo.

En cuanto al desdoblamiento base de este pensamiento musical distinguimos dos estéticas distintas, según la importancia que se le dé al contenido respecto a la forma. Por un lado, en el movimiento de carácter intramusical, que se manifiesta en la direccionalidad objeto-sujeto, podemos percibir la tendencia a proceder mediante similitud, ganando, según Stravinski, solidez (Ares Yebra, 2017). Por otro lado, en el movimiento extramusical prima la variedad y el contraste. Para el com- 
positor ruso, este contraste no hace más que diseminar la atención de los oyentes, aunque no rechaza la necesidad de variación.

Este principio, que en Souvtchinski se muestra como desdoblamiento y concentración, Stravinski lo recoge en su Poética distinguiendo contraste y similitud, siguiendo la presentación de ideas en forma de dualidad mediante distinciones dialécticas (Ares Yebra, 2014).

\section{La Suite Italiana}

La Suite italiana de Stravinski surge como una transcripción del ballet Pulcinella (1920) al formato de dúo de violín y piano. El ballet está escrito para orquesta de cámara y tres cantantes solistas. Derivado de él, podemos encontrar la Suite Pulcinella para orquesta, que data de 1922 y en la que el número de piezas se reduce a 8 , habiendo eliminado las partes cantadas. Por último, en la obra escogida para nuestro estudio, este número de movimientos se ve reducido, siendo estos Introducción, Serenata, Tarantella, Gavotte con variaciones, Minueto y Finale, en la primera edición.

El ballet Pulcinella está inspirado en un personaje homónimo de la Comedia del arte, una muy importante aportación del teatro italiano en el barroco y caracterizado por ser de carácter cómico e improvisatorio. Otro rasgo muy destacado es que sus personajes llevan máscaras y hacen gran uso de la mímica. Concretamente, este ballet deriva de un manuscrito de 1700 encontrado en Nápoles, del episodio titulado Quatre Polichinelles semblables (cuatro Pulcinellas idénticos).

Este ballet, como ya hemos explicado anteriormente, es una pieza clave dentro de la praxis compositiva de nuestro objeto de estudio, ya que marca el comienzo del periodo neoclásico del autor. En él, se pone de manifiesto la concepción del compositor de historia como permanencia, usándola como herramienta en su totalidad y en su continuidad (Boucourechliev, 1982). Sin embargo, no debemos dejar de lado la crítica negativa que recibió la obra, a la que se la calificó como pastiche.

El neoclasicismo supone un aumento en el interés por las épocas musicales pasadas, como es el barroco en el caso de Pulcinella. El propio Stravinski narra en Crónicas de mi vida la influencia que tuvieron las obras de Giovanni Pergolesi en la creación de este ballet. No obstante, siempre señala que la música de su periodo neoclásico no es una vuelta completa al pasado, sino una vista al reflejo de sí mismo.

Podemos observar este interés por él en la totalidad de la composición. Comenzando por la obra en general, destacamos que se trata de una composición al estilo de la suite barroca, ya que está dividida en movimientos de danza como el Minueto o la Gavota, ordenados de manera contrastante en cuanto a carácter. Hace uso de algunas melodías y líneas de bajo de las obras originales de Pergolesi, 
aunque Stravinski no deja de lado su personalidad compositiva, ya que también podemos encontrar recursos propios del compositor y de su estilo. Dichos recursos son, por ejemplo, la utilización de quintas paralelas que aparecen tanto en el violín como en el piano, la técnica de la nota equivocada, rearmonizaciones de la melodía con distintas sonoridades o tratamientos rítmicos diferentes, ofreciendo una manipulación de las ideas del siglo XVIII (Dorf, 2012).

Debemos destacar también la clasificación de la Suite italiana como transcripción de ballet, hecho bastante inusual hasta entonces. Pocos son los compositores que habían recurrido a esta opción, siendo sin embargo muy populares dentro del repertorio violinístico romántico las transcripciones de ópera, como por ejemplo Fantasía sobre temas de Fausto de Henryk Wieniawsky. No obstante, durante la vida de Stravinski las transcripciones de ballet se abren cierto paso dentro del repertorio del instrumento. De esta manera, encontramos, por ejemplo, las piezas del ballet Romeo y Julieta de Serguéi Prokofiev transcritas para violín y piano, así como transcripciones de otros ballets de Stravinski como El beso del Hada o piezas del Pájaro de Fuego.

\section{Las Transcripciones}

Podemos encontrar una primera edición de la Suite italiana que data de 1925 y está dedicada a Paul Kochanski, violinista polaco. Esta recibe el nombre de Suite para violin y piano sobre temas, fragmentos y piezas de Giambattista Pergolesi. Fue editada por Albert Spalding, y actualmente está en desuso. La edición que más se conoce de la obra es la de 1933, ya con el título de Suite italiana, en la que además de los cinco movimientos descritos se añade un Scherzino proveniente del ballet. Además de la inclusión de este nuevo movimiento, las diferencias entre ambas ediciones son significativas, cambiando en diversos puntos el tratamiento tanto del violín como del piano. En el caso del violín, se añade mayor variedad de recursos, como armónicos, pizzicatos y distintos golpes de arco (White, 1981). También se cambian aspectos de la edición anterior que causaban problemas en la ejecución o que resultaban incoherentes. Cabe señalar que Stravinski pretendía mantener su personalidad compositiva al mismo tiempo que respetar las limitaciones del instrumento, ya que el aspecto visual de la interpretación, como ya hemos mencionado, tiene cierta importancia para el compositor.

En la edición de 1925 podemos destacar diferentes técnicas del violín que dificultan en cierta medida su ejecución. Indica, así, dinámicas poco realistas respecto al golpe de arco que se pide. Respecto a la técnica de la mano izquierda, se destacan usos del pizzicato ejecutado con esta mano, que no dan especialmente buen resultado por el tempo al que se requiere el movimiento. Existen más problemas relacionados con el uso de las dobles cuerdas, ya que en esta edición observamos fragmentos en los que el uso de dicho recurso se explota tanto, que torna 
la ejecución más complicada. Debemos señalar que, antes de la intervención de Samuel Dushkin, la escritura de Stravinski para el violín era predominantemente percusiva, y hacía uso de diversos recursos del instrumento para llegar a tal fin, como es el caso de sucesiones de acordes o notas continuamente arco abajo (es decir, moviendo el arco desde el talón hacia la punta). Esta edición raramente se interpreta debido a su escritura poco idiomática (Seeger, 2015).

Dicha segunda edición tuvo la intervención de Dushkin, violinista de origen polaco con el que coincidió Stravinski en Estados Unidos en torno a la composición de su concierto para violín. Durante la creación del concierto, Dushkin fue de vital importancia para el compositor, ya que este poseía un conocimiento del instrumento bastante limitado. De esta forma, Dushkin le ofreció una visión más amplia y completa de lo que el violín puede hacer técnicamente, así como también cómo debería ser la escritura de la parte del instrumento principal para facilitar su correcta ejecución, todo esto sin renunciar a la estética del compositor. De esta manera, se marcó un antes y un después en la escritura del compositor para dicho instrumento. Stravinski no persigue la transcripción sencilla y de fácil ejecución, sino que mantiene la misma frescura y espíritu del original (White, 1981).

En cuanto a la figura de Dushkin, es muy importante conocer que fue alumno de dos grandes violinistas y pedagogos del instrumento, como fueron Leopold Auer y Fritz Kreisler. Este último es además conocido por sus transcripciones para violín y piano de obras de diferentes compositores entre los que encontramos a Frédéric Chopin, Isaac Albéniz, Manuel de Falla, Arcangelo Corelli, Aleksandr Glazunov, etc.

A continuación, vamos a proceder al análisis de dos números del ballet en comparación con el correspondiente en la Suite italiana.

\section{Análisis de los movimientos escogidos}

\section{Gavotta con variazioni}

Podemos observar en este número del ballet que la sección de cuerda no aparece; es un número exclusivo de viento. Debido a esto, destacamos en el número correspondiente de la Suite italiana el uso variado de los diferentes timbres que puede producir el violín y, para ello, el uso de las diferentes técnicas que los hacen posible. A continuación, vamos a detallar de qué instrumentos del ballet Pucinella toman Stravinski y Dushkin los materiales para la parte de violín de la Suite italiana, comparando también las posibles diferencias en ambas escrituras. Para ello, hemos usado las partituras del ballet y de la Suite italiana de la editorial Boosey \& Hawkes 0. En el caso del ballet, la edición usada es la revisión de 1965 (Stravinski, 1933, 1965). 
En el ballet, los 10 primeros compases corresponden al oboe, por ello se busca una sonoridad abierta y resonante, utilizando para ello posiciones bajas. En los compases 11-14, donde se ha transcrito la intervención de la flauta, se recurre a posiciones más altas en cuerdas más graves, ofreciendo así una sonoridad aterciopelada y más suave, además de indicar la dinámica piano. El compás 15 vuelve a ser parte de la intervención del oboe, por lo que se indica una dinámica mezzopiano y se vuelve a la sonoridad abierta. A diferencia de la edición anterior, todo el material melódico queda relegado al violín, mientras que al piano se le otorga el contenido armónico (Tu, 2012). En el compás 25 toma el material de la trompa 1 por dos compases, para lo cual recurre a una sonoridad más "oscura". En los siguientes compases el violín interpreta las líneas del fagot 1 y otros instrumentos que aportan la armonía, retomando el papel de la flauta 1 en el compás 29.

En cuanto a las diferencias en la escritura y articulación en esta sección, lo más destacable es el compás 27, en el que podríamos entrever la intervención de Dushkin, ya que articula la nota tras el armónico para asegurar su sonoridad (ver Figura 1).

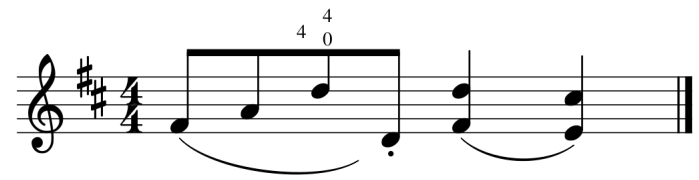

Figura 1. Suite taliana. Gavotta con variaz̧ioni, compás 27. (Stravinski, 1933).

En la primera variación, la línea que recoge el violín es la del oboe 1, alternándose en los compases 19 a 24 con el oboe 2. También toma alguna pequeña intervención de la trompa 1, como es el caso del compás 10. En ella, podemos ver más diferencias en cuanto a la articulación, ya que desde el comienzo es completamente distinta. En el oboe, Stravinski había empleado ligaduras más largas, mientras que en la transcripción predomina una articulación más separada. Esta diferencia podría deberse a una facilitación para el violinista a la hora de interpretar la partitura, ya que mejora la consecución del carácter requerido por la pieza. También se añade algún ornamento que no aparece en el ballet, como es el caso de la apoyatura inicial.

A partir del compás 11 se añade una diferencia más, ya que la línea del oboe se ve transportada a la octava superior, obteniendo así un sonido más brillante en el violín y haciéndolo destacar más. Entre los compases 19 y 24 cabe destacar una diferencia de articulación que nos ha llamado la atención, ya que en el original el oboe ejecuta las notas picadas, con escasas ligaduras, mientras que en el caso de la 
transcripción se mantiene el motor de articulación que venía predominando en la variación (de tres corcheas, dos ligadas y una suelta) (ver Figura 2).

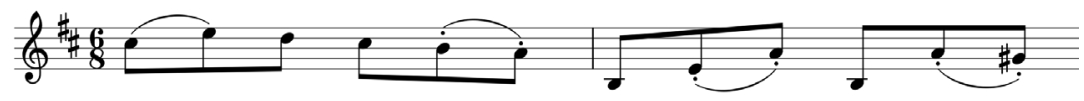

Figura 2. Suite italiana. Gavotta con variaz̨ioni, primera variación. Compases 8 y 9. (Stravinski, 1933).

La segunda variación alterna las líneas melódicas de la flauta 1 y la trompa 1 , llegando a ejecutarse al mismo tiempo en los compases 4 y 5. Cuando el violín realiza la parte de la flauta, se han indicado en este caso posiciones más bajas, obteniendo así una sonoridad más abierta; mientras que cuando interviene la trompa se indican posiciones más altas y cuerdas más graves, favoreciendo así una diferencia tímbrica en el violín. Cabe destacar el caso de los compases 4 y 5 , donde se unen las dos líneas. En el compás 4 podemos observar que se aprovecha el recurso de la cuerda al aire, en este caso la primera cuerda $M i$, facilitando así la ejecución, mientras que, a partir de la última corchea de ese mismo compás, la línea de la trompa se indica en la octava inferior, que es donde debería haberse situado (ver Figura 3).

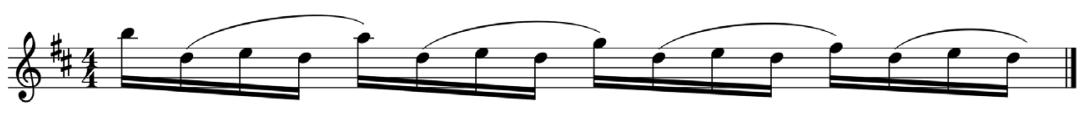

Figura 3. Suite italiana. Gavotta con variazioni, segunda variación. Compás 4. (Stravinski, 1933).

Una diferencia notable en la transcripción respecto al ballet es también la ausencia de la repetición del compás 9. Así, en la versión de violín y piano no podremos observar casillas de repetición, ejecutándose directamente lo que correspondería a la segunda casilla en el ballet. Seguimos observando diferencias en las ligaduras, posiblemente por las diferentes cuestiones técnicas de los instrumentos. La mayor de estas diferencias la encontramos en los dos últimos compases de la variación (ver Figura 4a y b):

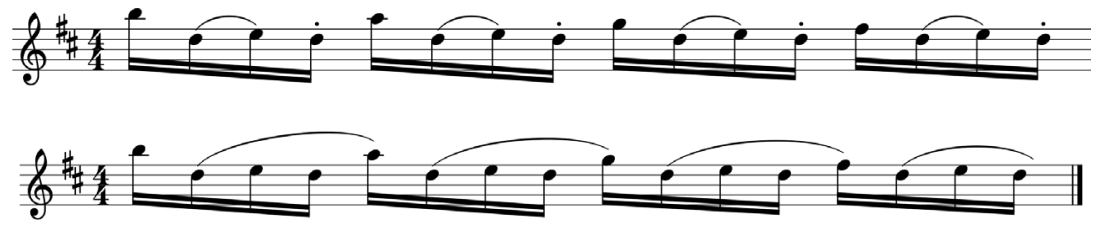

Figura 4: a. Ballet Pulcinella. Gavotta con variazioni, segunda variación. Compás 15. (Stravinski, 1965);

b. Suite italiana. Gavotta con variaz̧oni, segunda variación. Compás 15. (Stravinski, 1933). 
También destacamos que, en el caso del violín los grupetos suelen aparecer coincidiendo con el inicio de una nueva ligadura, lo que facilita su correcta ejecución, y donde podríamos suponer también la intervención de Dushkin.

\section{Finale}

Este movimiento es significativamente complejo. En él, Stravinski emplea la orquesta del ballet al completo, por lo que tenemos más voces para utilizar en la transcripción. Debemos señalar también que en Pulcinella este movimiento está indicado como Allegro assai. Como consecuencia, los cambios de instrumento que recoge la línea del violín en la Suite italiana son constantes, por lo que vamos a comentarlos simultáneamente a las diferencias encontradas para facilitar su seguimiento.

En el primer compás ya existe un elemento distinto, puesto que la escala ascendente no aparece en ningún instrumento en el ballet, siendo así un adorno de la transcripción. Los siete primeros compases están tomados de la flauta 1 y el violín 1, pero en la octava inmediatamente inferior. Podemos observar diferencias de articulación incluso siendo el mismo instrumento. Así, en el ballet, Stravinski indica a los violines detaché y a la flauta, lágrimas, mientras que en la Suite italiana lo que observamos son acentos.

Entre el segundo pulso del compás 7 y la caída del compás 11, el compositor toma las líneas de la trompeta y la trompa 1, aprovechando las capacidades técnicas del violín. La articulación es distinta, ya que en los instrumentos de viento predominan las figuras sueltas con punto y un apoyo en la primera nota de cada compás, mientras que en el violín se presenta lo siguiente (ver Figura 5):

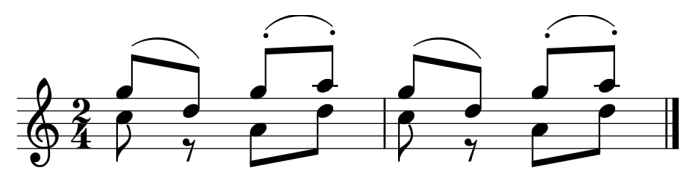

Figura 5. Suite italiana. Finale. Compases 8 y 9. (Stravinski, 1933).

Esta diferencia puede deberse a una adaptación de lo requerido por el compositor en el ballet a la técnica instrumental del violín, ya que al hacer esos arcos se favorece que la primera nota de cada compás cada compás caiga arco abajo, y, por lo tanto, se produzca un apoyo natural. En el compás 11, el violín realiza las voces de la viola y los violines, dando como resultado una escala por quintas descendentes, algo inusual para el instrumento. En los siguientes tres compases aparece un pequeño material que no existe en el ballet.

Entre los compases 15 y 20, mientras que el piano toca el solo de la flauta 1 
y un acompañamiento también nuevo, el violín toma el papel de la flauta 2 en octavas para completar la sonoridad. Llama la atención que, cada dos compases, la nota que produce el trino coincide con la nota que está tocando la trompa 2.

Además, tenemos que señalar que la ausencia de ligaduras en el violín en la transcripción es una ayuda para ejecutante, ya que deja un espacio para ejecutar el movimiento correcto de la mano izquierda (Tu, 2012).

Del compás 21 al 29 toma la línea de los violines primeros, especialmente del solista. Hay algunas diferencias, entre las cuales señalamos la ligadura del compás 21, que permite que se llegue del spiccato a una zona más próxima al talón para comenzar el tema del compás 22. Apreciamos también diferencias en las dinámicas, ya que en el ballet existen indicaciones que se omiten en la transcripción. El compás 30 pertenece a los violines segundos en el ballet, y a partir de ahí va alternando las dos partes hasta ejecutar la voz superior de ambas simultáneamente a partir del compás 35. Nos llama la atención la indicación leggero en la transcripción (compás 36), ya que en su lugar en el ballet aparece du talon. Al mismo tiempo, hay articulaciones ligeramente diferentes en ambas partituras, ya que en el ballet se indican lágrimas y en la suite puntos, siendo el mismo instrumento en ambos casos.

Los acordes de los compases 41 al 43 se corresponden en el ballet con las voces de violines primeros y viola, mientras que los siguientes dos compases y la caída del tercero serían las de violines primeros y segundos, aunque únicamente toma los sforzando, que corresponden a su vez con la figuración que podemos ver en el viento. En el segundo pulso del compás 46 se han transcrito los acordes correspondientes a los fagotes y la trompa 1. Debemos señalar que en la transcripción no hay indicaciones de articulación a excepción del acento en la primera de las semicorcheas, mientras que en el ballet dicho acento no aparece y hay indicaciones de lágrimas en los fagotes. No obstante, no se pierde el carácter marcado que indica Stravinski en Pulcinella, ya que se han escrito arcos abajo consecutivos en las corcheas, obteniendo así una sonoridad más percusiva.

En el compás 49 podemos observar que la última corchea ha sido omitida presumiblemente para asegurar y facilitar la ejecución del siguiente compás, donde el violín toma momentáneamente el papel de trompeta subido una octava para completarlo con cuerdas al aire, aumentando su sonoridad. Esta intervención es muy corta, ya que el papel de la trompeta continúa en el piano en ese mismo compás en su octava original, mientras que el violín lo deja y toma el de los violines primeros en el siguiente compás, hasta el 55. Aquí, pese a ser el mismo instrumento que el original, hay diferentes indicaciones, ya que en el ballet no aparecen ni acentos ni puntos. Como consecuencia de lo requerido por el compositor en la transcripción, se indica tocar arcos abajo consecutivos, facilitando así el carácter y la obtención de los acentos (ver Figura 6). 


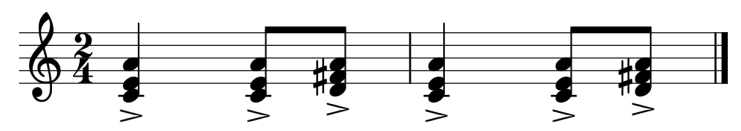

Figura 6. Suite italiana. Finale. Compases 53 y 54. (Stravinski, 1933).

Del segundo pulso del compás 55 a la caída del 59 utiliza el mismo recurso que había empleado entre el 7 y el 11, permaneciendo el compás de la caída con la voz de la trompa. Los acordes que aparecen en los siguientes compases se corresponden con los de la cuerda en el ballet, pero con las notas reordenadas para adecuarlos a un solo instrumento. Desde la anacrusa del compás 63 toma la línea del violín 1 solo. Esta es muy llamativa, ya que se indican ligaduras y digitaciones completamente diferentes (ver Figuras $7 \mathrm{a}$ y b).

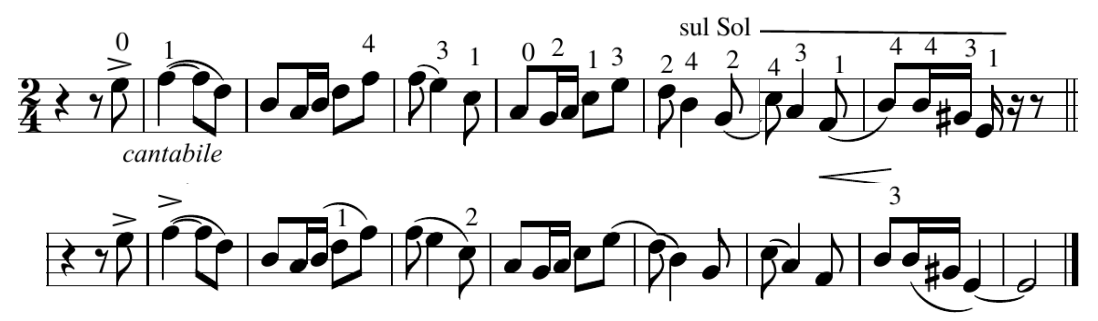

Figura 7: a. Pulcinella. Allegro assai. Compases 63 al 69. (Stravinski, 1965).; b. Suite italiana. Finale. Compases 63 al 70. (Stravinski, 1933).

La siguiente intervención corresponde a los primeros violines. Los pizzicatos de los compases 75-76 y 79-80 llaman nuestra atención por ser los intervalos que conforman las trompas, pero con la figuración de la cuerda baja. Entre ellos, toma el solo de la trompeta con cuerdas al aire que aumentan la sonoridad. En el compás 80 toma la voz de los violines segundos, sumándose los primeros entre el 82 y el primer pulso del 84 . Los acordes de 86 y 87 son los que ejecutan las trompas, mientras que los siguientes se corresponden a los primeros violines, que se mantienen hasta el compás 94. Aparecen aquí algunas diferencias de articulación, como son los acentos presentes en 89 en el ballet e inexistentes en la Suite italiana. En los siguientes compases encontramos un elemento que no aparece en el original: las tres semicorcheas iniciales de cada compás. El resto se corresponde en 95 con la trompa 1 y en 96 con trompeta, violín 2 y viola una octava por debajo.

El material entre los compases 99 y 103 se corresponde con el solo de flauta 1 una octava más grave de su original, adaptándose así a una tesitura más cómoda para el violín. La escala ascendente que le sigue se toma de la parte de violines solos, con una pequeña diferencia de arco en 104 que facilita la caída en el siguiente compás, al igual que había ocurrido en 22. Hasta el compás 113 se toma el solo del 
primer violín. Los siguientes acordes se corresponden con el inicio de cada célula, reforzando así el desplazamiento del ritmo que se produce.

A partir del 117 surgen muchas diferencias entre Pulcinella y la Suite italiana. Principalmente toma las voces de trompa 1 y trompeta, con alguna intervención de la cuerda como es el caso del compás 125. En el solo de trompa con el que comienza recurre a la cuarta cuerda Solpara obtener una sonoridad más adecuada, completando la sonoridad con cuerdas al aire y acordes que le otorgan al violín una mayor sonoridad, adecuada para el carácter de esta sección.

En el compás 121 de Pulcinella se introduce un compás de 3/4 que no aparece en la Suite italiana, por lo que se causa un descuadre en la música de ambas partituras. No obstante, se mantiene la misma dinámica mencionada anteriormente, tomando principalmente los papeles de trompa 1 y trompeta, mientras que el piano se mantiene en un ostinato rítmico hasta el final.

Debemos destacar que a partir del 123 se produce una diferencia respecto a la Suite sobre temas, fragmentos y piezas de Giambattista Pergolesi, ya que en ella el solo aparecía en la octava inferior, dando como resultado una mayor dificultad técnica y menor brillantez en el sonido (Tu, 2012). Así, podemos apreciar la gran importancia e influencia que tuvo Dushkin para transformar la Suite italiana en una obra idiomática e importante dentro del repertorio violinístico.

\section{Conclusiones}

La estética de Stravinski es un campo muy amplio e interesante, en el que se pueden estudiar diversos aspectos de gran importancia para la comprensión de su música. Es muy importante la correcta comprensión de su estética para entender sus obras, ya que especula sobre el fenómeno musical, en gran medida, otorgando un papel muy importante al tiempo y al ritmo. Así, al acercarnos más a cómo se articula su pensamiento, podemos ejecutar con más corrección sus obras. El intérprete para Stravinski es una figura clave para la transmisión de lo escrito por el compositor sin espacio para la subjetividad, de ahí que debamos tener una lectura muy precisa y rigurosa de su escritura. Debemos recordar que para él la música es una organización del tiempo, y que procede mayoritariamente por similitud.

Destacamos la gran importancia de su pensamiento estético y cómo lo diferencia claramente de los compositores contemporáneos a él, haciéndolo resaltar tanto en el ámbito teórico como en el compositivo. En cuanto al análisis, hemos podido observar la gran intervención que tuvo Dushkin a la hora de hacer de la transcripción del ballet Pulcinella una obra más accesible al instrumentista, y por lo tanto más idiomática, aprovechando los diferentes recursos técnicos del violín 
a su favor. Lo podemos comprobar en las distintas articulaciones, los cambios de octava, la explotación de las cuerdas al aire, entre otros recursos.

También se destaca la importante disminución que se aprecia en sus anotaciones, hecho que nos resalta la etapa neoclásica de Stravinski como un periodo en el que su estética cambia de manera significativa.

Cabe señalar el atractivo que tiene poder comparar el ballet con su transcripción, detallando de dónde proceden los diferentes elementos. Este recurso resulta muy útil para la interpretación de la pieza, ya que con este conocimiento puede hacerse con mayor rigurosidad.

No obstante, con este artículo se ha establecido un comienzo para lo que consideramos una línea importante de investigación respecto a la figura de Stravinski. La Suite italiana merece un estudio más detallado y pormenorizado de sus componentes, siendo de bastante interés tanto lo práctico como lo teórico. Además, consideramos importantes otro tipo de estudios en cuanto a la figura del compositor, como puede ser la comparación de obras de diferentes etapas para analizar cómo se desarrolla su estética.

\section{Referencias}

Ares Yebra, J. (2017). Pierre Sowvtchinsky: desdoblamiento y concentración en Igor Stravinsky. Fuentes y elementos para una propuesta [tesis doctoral, Universidad de Vigo]. Repositorio Institucional Investigo.

Ares Yebra, J. (2014). El manuscrito de Souvtchinsky y la Poética musical: sobre el concepto de tiempo en el pensamiento estético de Igor Stravinsky. En Y. Espiña \& M. Ribeiro-Pereira (coord.), Arte \& Teoría. Cadernos de Teoría das Artes, 3 (pp. 95-121). Universidade Católica Editora.

Boucourechliev, A. (1982). Igor Stravinsky (D. Zimbaldo, trad.). Editorial Turner.

Craft, R. (1971). Stravinsky. Ideas y recuerdos (J. Godo, trad.). Aymá Sociedad Anónima Editora.

De la Motte, D. (1995). Contrapunto (M. Centenero, trad.). Editorial Labor.

Díaz Soto, D. (2009). Formalismo y materialismo. El problema de la materialidad del arte en Eduard Hanslick. Bajo palabra. Revista de Filosofia. Época no II, 4, 173-180. http:// www.bajopalabra.es/numeros-anteriores/epoca-n-ii-n-3-ano-2009/item/279-formalismo-y-materialismo-el-problema-de-la-materialidad-del-arte-en-eduard-hanslick 
Dorf, S. (2012). Review: Stravinsky's 'Pulcinella': A Facsimile of the Sources and Sketches; Nijinsky's Bloomsbury Ballet: Reconstruction of the Dance and Design of 'Jeux' [reseña del libro Stravinsky's 'Pulcinella": A Facsimile of the Sources and Sketches editado por M. A. Carr, y del libro Nijinsky's Bloomsbury Ballet: Reconstruction of the Dance and Design of 'Jeux', de M. Hodson]. Journal of the American Musicological Society, 65(1), 272-284. https://doi.org/10.1525/jams.2012.65.1.272

Ferrer, R. (2012). El concepto de evolución estética en Stravinsky. Artseduca, 2, 28-37.

Fubini, E. (2005). La estética musical desde la Antigüedad hasta el siglo XX. Alianza Editorial.

Imberty, M. (2001, 5 de mayo). Nuevas perspectivas en Psicología de la Música. La problemática del tiempo continuo y del tiempo discontinuo en la música del siglo XX (B. Sánchez \& J. Casabellas, trad.). Primera Reunión anual de la Sociedad Argentina de las Ciencias Cognitivas de la música, Buenos Aires, Argentina. http://saccom.org.ar/blog/ actas-de-congresos /

Kaiero, A. (2007). Creación musical e ideologías: la estética de la postmodernidad frente a la estética moderna [tesis doctoral, Universidad Autónoma de Barcelona]. http://hdl.handle. net $/ 10803 / 5197$

Manufacturing Intellect. (2020, 21 de agosto). Igor Stravinsky interview with Robert Craft (1957). [video]. You'Tube. https://youtu.be/JPH2Im-7nwo

Olives, J. (2004). Para una filosofía de la música. Un enfoque fenomenológico. Aproximación al pensamiento de Ernest Ansermet [tesis doctoral, Universidad de Barcelona]. Repositorio de la Universidad de Barcelona. http://diposit.ub.edu/dspace/handle/2445/58286

Romeu, V. (2007). Arte y comunicación: apuntes para una reflexión sobre la comunicación artística. Anuario CONEICC de Investigación de la Comunicación, 15.

Ruiz Molina, D. (2018). El arte definido como acto comunicativo. Revista AV Notas, 6, 111- 123. http://publicaciones.csmjaen.es/index.php/pruebas/article/ view/184/155

Seeger, L. (2015). Stravinsky and the Violin: Aspects of Style in Stravinsky's Violin Transcriptions [tesis doctoral, Universidad Estatal de Michigan].

Stravinski, I. (1946). Poética musical. (Grau, E. trad.) Editorial Emecé.

Stravinski, I. (1933). Suite Italienne, violin and piano. Editorial Boosey \& Hawkes.

Stravinski, I. (1936). Crónicas de mi vida. (García-Pérez, J. trad.) Ediciones de Nuevo Arte Thor.

Stravinski, I. (1965). Pulcinella Ballet. Editorial Boosey \& Hawkes. 
Tu, K. (2012). In the Fingertips: RONI a Discussion of Stravinsky's Violin writing in bis Ballet Transcriptions for Violin and Piano [tesis doctoral, Universidad de Cincinnati]. Repositorio Electronic Theses \& Dissertations Center. http://rave.ohiolink.edu/etdc/ view?acc num $=u c i n 1337288174$

White, K. (1981). Violin works of Igor Stravinsky [tesis doctoral, Universidad de Arizona]. Repositorio de la Universidad de Arizona. http://hdl.handle. net/10150/624866 